\title{
Comparaison du comportement hydrique et de la capacité photosynthétique du maïs et du tournesol en condition de contrainte hydrique. Conclusions sur l'efficience de l'eau
}

\author{
N Katerji, O Bethenod * \\ Unité de bioclimatologie, Inra, F-78850 Thiverat-Grignon, France
}

(Reçu le 29 janvier 1996 ; accepté le 29 novembre 1996)

\begin{abstract}
Summary - A comparison of water and photosynthesis behaviour of corn and sunflower under water stress. Inference on water use efficiency. Various indices of water stress sensitivity lead to contradictory conclusions as to the choice of irrigated crop between corn and sunflower. We propose an additional analysis of leaf gas exchanges after flowering to field analysis on dry matter and crop water use. Gas exchanges indicated that soil water depletion induces stomatal closure in both plants. For corn. this stomatal closure was accompanied by a reduction in photosynthesis when predawn leaf water potential decreased below $-0.2 \mathrm{MPa}$. Without water stress, stomatal conductance and transpiration were between two and three times higher for sunflower than for corn. The reduction in leaf photosynthesis in sunflower began at a predawn leaf water potential below - $0.5 \mathrm{MPa}$. For a given value of stomatal conductance, corn leaf photosynthesis was always higher than that of sunflower. Complementary information on physiological mechanisms at leaf level was derived; this should contribute to a better irrigation efficiency at field level.
\end{abstract}

corn / sunflower / photosynthesis / transpiration / water stress

Résumé - Les différents indices de sensibilité à la sécheresse conduisent à des conclusions contradictoires pour le choix des cultures irriguées entre le maïs et le tournesol. Nous proposons de compléter les analyses effectuées au champ sur la matière sèche et la consommation en eau des cultures par l'observation des échanges gazeux foliaires après floraison. Ceci montre qu'une diminution des réserves hydriques du sol provoque chez les deux plantes la fermeture stomatique. Chez le maïs, cette fermeture stomatique s'accompagne d'une diminution de la photosynthèse à partir d'un potentiel de base de -0,2 MPa. Chez le tournesol, dont les valeurs maximales de conductance stomatique et de transpiration sont deux à trois fois supérieures à celles du maïs en l'absence de contrainte hydrique, la diminution de photosynthèse n'apparaît qu'à partir d'un potentiel de base de -0,5 MPa. Pour une valeur donnée de conductance stomatique, la photosynthèse des feuilles de maïs est toujours supérieure à celle du tournesol. Les conclusions apportent des bases complémentaires de raisonnement pour réaliser une meilleure efficience de l'eau apportée.

Zea mays = maïs $/$ helianthus annuus $=$ tournesol $/$ photosynthèse $/$ transpiration $/$ contrainte hydrique

* Correspondance et tirés à part.

Tél (33) 01 30815555 ; fax : (33) 0130815563 


\section{INTRODUCTION}

La culture du tournesol a connu au cours des dernières années une extension importante dans les pays de l'Europe du Sud. La raison principale est que cette culture a été considérée comme alternative à la culture du maïs lorsque la sécheresse est prévisible. À titre d'exemple, les surfaces irriguées en tournesol ont été multipliées par 90 entre 1979 et 1990 et par deux entre 1988 et 1990 (Lesaffre et Rieul, 1992).

Le choix du tournesol comme alternative au maïs revient à admettre que le tournesol est plus résistant à la contrainte hydrique. Ce constat est cependant un sujet de controverse dans la littérature (cf la revue bibliographique de Robinson, 1978). Les premiers travaux laissent supposer en effet que le tournesol gaspille l'eau alors que le maïs est plutôt économe (Shantz et Piemeisel, 1927; Barnes, 1938; Mihalyfalvy, 1962). Ces auteurs ont noté que le tournesol consomme $40 \%$ de plus au cours du cycle végétatif alors que l'efficience de l'eau consommée est deux fois (cas de la production de la matière sèche) à trois fois inférieure (si on considère la production en grain) à celle du maïs. Ces comparaisons doivent être cependant nuancées, car le cycle de la culture est plus long chez le tournesol et la composition des grains est différente. Ceux du maïs renferment principalement de l'amidon alors que ceux du tournesol contiennent $50 \%$ d'huile et $20 \%$ de protéines. Or, le coût en glucose de la biosynthèse des lipides et des protéines est supérieur à celui de l'amidon (Blanchet et al, 1982 ; Penning de Vries et al, 1974). Les résultats donnés par ces derniers auteurs permettent de calculer qu'à partir de $1 \mathrm{~g}$ de glucose on peut obtenir soit $0,62 \mathrm{~g}$ de protéines, soit $0,35 \mathrm{~g}$ de lipides.

Le tournesol a été considéré comme une culture résistante à la sécheresse à la suite des travaux de Puech et al (1976) et de Doorenbos et Kassam (1979).

Ces auteurs avaient analysé les valeurs relatives en matière sèche et en grains ( $\mathrm{Ms}$ ) et de consommation en eau (ETR) par rapport au rendement maximal (Msm) supposé coïncider avec l'évapotranspiration maximale (ETM) pour plusieurs espèces végétales. Les résultats trouvés paraissent s'ajuster à une relation linéaire de la forme suivante :

$$
\frac{M s}{M s m}=a \frac{E T R}{E T M}+b
$$

Dans cette relation, le coefficient $a$ peut être considéré comme un indice de sensibilité à la sécheres- se : plus il est élevé, plus la culture est sensible à celle-ci. On a noté alors que le coefficient $a$ est nettement plus petit chez le tournesol que chez le maïs. Ce dernier était considéré sensible au déficit hydrique alors que le tournesol était classé résistant. Cette classification a été dernièrement remise en question pour différentes raisons :

- la valeur du coefficient $a$, chez le tournesol, semble varier considérablement selon le niveau de l'ETM (Texier, 1992) ;

- on a observé une variabilité importante du rendement (pratiquement du simple au double) dans des situations où le rapport ETR/ETM était pourtant identique (Merrien et Grandin, 1990) ;

- dans le cadre des études réalisées sur la réponse du tournesol et du mais à la contrainte hydrique d'origine saline, il apparaît que le tournesol et le maïs présentent une sensibilité identique (Katerji et al, 1996).

Pour tenter d'éclairer le débat, il nous a semblé utile d'envisager une voie d'analyse autre que celle du comportement agronomique fondé sur les notions de consommation en eau et de rendement. La voie que nous envisageons est fondée sur l'analyse du comportement hydrique et photosynthétique chez le maïs et le tournesol en condition de contrainte hydrique et ses répercussions sur l'efficience instantanée de l'eau sur une période d'un mois pendant et après la floraison des plantes. D'une façon plus précise, nous proposons :

- d'analyser les conséquences de la contrainte hydrique d'origine édaphique sur l'état hydrique et l'ouverture stomatique.

- de comparer la capacité photosynthétique du maïs et du tournesol sous différents régimes hydriques.

- d'analyser les conséquences sur l'efficience de l'eau en se fondant sur la théorie de l'offre et la demande proposée par Jones (1973).

La confrontation entre les démarches agronomiques et physiologiques permettra d'éclairer davantage l'aptitude des espèces étudiées à produire en condition de contrainte hydrique. De cette confrontation découleront également des raisonnements qui contribueront à réaliser une meilleure efficience de l'eau apportée.

\section{MATÉRIEL ET MÉTHODES}

Les études ont été réalisées avec des cultures de plein champ sur le site expérimental de l'unité de recherche en bioclimatologie de Grignon au cours des années 1990 et 1992, pendant des journées ensoleillées. 
Le maïs (Zea mavs L cv LGl) a été semé le 3 mai 1990 et la floraison a été atteinte le 12 juillet. À cette date, l'indice foliaire était de 3,2 et la densité était de $9 \mathrm{pl} \cdot \mathrm{m}^{-2}$.

Le tournesol (Helianthus annuus L cv Albena) a été semé le 22 avril 1992. À la floraison, le 10 juillet, l'indice foliaire était de 4,3 et la densité était de $8,4 \mathrm{pl} \cdot \mathrm{m}^{-2}$.

Les observations concernant le comportement hydrique et photosynthétique ont été réalisées pendant la semaine de floraison et les 3 semaines suivant cette date. Pendant cette période, les cultures n'ont reçu aucune irrigation. Au cours de cette période, le maïs et le tournesol présentent une sensibilité accrue à la contrainte hydrique (Robelin, 1967) et la conductance stomatique varie peu avec le stade végétatif (Bethenod et al, 1982).

\section{Caractérisation de l'état hydrique et des échanges gazeux}

L'état hydrique de la plante a été caractérisé à partir des mesures du potentiel hydrique foliaire. Cette grandeur a été mesurée à la fïn de la nuit (potentiel de base) et à 12 heures (heure solaire). Six feuilles ont été prélevées à chaque fois et leur potentiel a été mesuré au moyen d'une chambre à pression.

La conductance stomatique des faces supérieures et inférieures de dix feuilles situées au sommet de la végétation a été mesurée à 12 heures au moyen du poromètre à diffusion Delta TIII. L'étalonnage du poromètre a été réalisé avant et après chaque série de mesure selon la procédure décrite dans Tardieu et al (1990).

Les mesures couplées de photosynthèse nette $\mathrm{A}_{n}$ et de la conductance stomatique à la diffusion du $\mathrm{CO}_{2}, \mathrm{~g}_{i}$, ont été effectuées à $8,10,12$ et 14 heures au moyen d'une chambre de Parkinson en circuit ouvert (LCA3, $\mathrm{ADC}$ ). Ces mesures ont été réalisées sur des feuilles bien éclairées, situées au sommet de la végétation.

L'étalonnage de la chambre de Parkinson a été mené, selon la méthode décrite par Bethenod et al (1988), avant et après chaque série de mesure. Dans une étude précédente (Tardieu et al, 1990), on a vérifié que les conductances stomatiques mesurées par le poromètre Delta TIII et la chambre de Parkinson sont très voisines.

Enfin, pour vérifier la cohérence entre les indicateurs caractérisant l'état hydrique de la plante et ceux relatifs au sol, nous avons suivi les variations du stock d'eau dans le sol au cours de l'année 1990. Elles étaient déterminées au moyen d'un humidimètre neutronique (Nardeux Solo 25). Six tubes d'accès de $2 \mathrm{~m}$ de longueur ont été placés à $0,1 \mathrm{~m}$ du rang de semis et 5 à 6 mesures par tube ont été périodiquement réalisées tous les $0,1 \mathrm{~m}$ de profondeur.

\section{Analyse de l'efficience de l'eau}

L'étude de l'efficience de l'eau s'appuie sur l'analyse du rôle respectif des composantes stomatiques et non stomatiques qui interviennent dans la variation de la photosynthèse. Jones (1973) sépare ces deux composantes en fonction de la concentration en $\mathrm{CO}_{2}$ des espaces intercellulaires $\left(\mathrm{C}_{i}\right)$ pour une concentration en $\mathrm{CO}_{2}$ de l'air $\left(\mathrm{C}_{a}\right)$ donnée. Le principe consiste à admettre une fonction d'offre qui exprime la photosynthèse $\left(\mathrm{A}_{n}\right)$ comme la densité de flux de $\mathrm{CO}_{2}$ qui traverse les stomates et la couche limite de la feuille :

$$
A_{n}=g_{c}\left(C_{a}-C_{i}\right)
$$

et une fonction de demande qui exprime la fixation de $\mathrm{CO}_{2}$ par le mésophylle :

$$
A_{n}=f\left(C_{i}\right)
$$

L'intersection des deux fonctions détermine les valeurs d' $\mathrm{A}_{n}, \mathrm{~g}_{i}$, et $\mathrm{C}_{i}$. Ceci constitue un moyen simple pour aborder cette efficience à l'échelle foliaire en distinguant les processus diffusifs en phase gazeuse des processus mésophylliens de la fixation du $\mathrm{CO}_{2}$ (processus non stomatiques). Trois situations sont alors envisagées lorsqu'on aborde cette relation (fig 1 ) :

- une diminution de $\mathrm{g}_{\mathrm{c}}$ sans modification de la relation entre $\mathrm{A}_{n}$ et $\mathrm{C}_{i}$, ce qui signifie que la conductance mésophyllienne à la diffusion du $\mathrm{CO}_{2}, \mathrm{~g}_{m}$, reste constante alors que $\mathrm{g}_{c}$ diminue. Il en résulte une augmentation de l'efficience de l'eau (fig 1, trajectoire $a$ ) ;

- une diminution de $\mathrm{A}_{n}$ sans modification de $\mathrm{g}_{c}$, ce qui signifie que $g_{m}$ diminue alors que $g_{c}$ reste constante. Il en résulte une diminution de l'efficience de l'eau (fig l, trajectoire $b$ ) ;

- une relation de proportionnalité entre $A_{n}$ et $g_{c}$, ce qui signifie qu'une diminution de la conductance mésophyllienne, $\mathrm{g}_{m}$, à la diffusion du $\mathrm{CO}_{2}$ provoque simultanément une diminution de la conductance stomatique, $\mathrm{g}_{\mathrm{c}}$. Il en résulte un maintien de l'efficience de l'eau (fig 1 , trajectoire $c$ ).

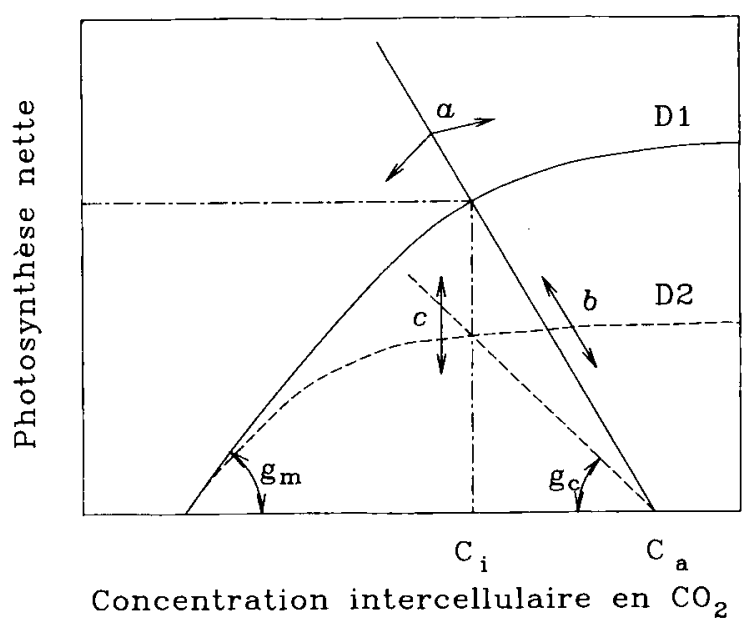

Fig 1. Schéma permettant d'analyser les facteurs limitants de la photosynthèse foliaire. La fonction de demande en $\mathrm{CO}_{2}$, illustée par $\mathrm{D}_{1}$ et $\mathrm{D}_{2}$, représente l'assimilation nette de $\mathrm{CO}_{2}$ en fonction de la concentration en $\mathrm{CO}_{2}$ des espaces intercellulaires calculée par les échanges gazeux. $\mathrm{g}_{m}$ représente la conductance mésophyllienne maximale et $\mathrm{g}_{\mathrm{c}}$ la conductance stomatique pour le $\mathrm{CO}_{2}$. Les modalités de régulation des échanges gazeux foliaires sont représentés par trois trajectoires différentes : $a$ : fonction de demande constante $; b$ : fonction d'offre constante ; $c$ : ajustement simultané des fonctions d'offre et de demande. 
Dans le plan $\left(\mathrm{A}_{\mu l}, \mathrm{~g}_{c}\right)$, le rapport de ces deux valeurs $\left(\mathrm{A}_{n} / \mathrm{g}_{1}\right)$ représente le gradient de concentration entre $\mathrm{C}_{a}$ et $\mathrm{C}_{i}$ (éqn. [1]). Les variations de $\mathrm{C}_{a}$ étant faibles sur l'échelle de temps considérée, toute diminution du rapport $\mathrm{A}_{\|} / \mathrm{g}$, représente une augmentation de $\mathrm{C}_{i}$ et une diminution de l'efficience de l'eau.

\section{RÉSULTATS}

\section{Comportement hydrique du maïs et du tournesol en condition de contrainte hydrique}

La figure 2 présente les données observées sur la culture de maiis : variation des réserves hydriques dans le sol, potentiel de base, potentiel hydrique foliaire et conductance stomatique à la diffusion de l'eau $\left(\mathrm{g}_{s}\right)$, mesurés à midi solaire. On constate d'abord qu'il y a une bonne concordance entre les évaluations des réserves hydriques dans le sol et les valeurs mesurées de potentiel de base et de conductance stomatique. En revanche, le potentiel hydrique minimal mesuré à midi présente une diminution beaucoup plus faible de $-1,7$ à $-2,1$ MPa. La conductance stomatique diminue rapidement de 5 à $2 \mathrm{~mm} \mathrm{~s}^{-1}$ lorsque le potentiel de base diminue de $-0,2$ à $-0,6 \mathrm{MPa}$ (jour 204) puis sa valeur fluctue entre 1 et $2 \mathrm{~mm} \mathrm{~s}^{-1}$, alors que le potentiel de base continue à diminuer de $-0,6$ à $-1,2 \mathrm{MPa}$.

La figure 3 présente les valeurs observées sur tournesol du potentiel de base, du potentiel hydrique foliaire et de la conductance stomatique, mesurés à midi. La diminution du potentiel de base est bien corrélée avec la diminution de la conductance stomatique. Le potentiel hydrique foliaire est plus sensible à la sécheresse que celui du maïs puisqu'il diminue d'une façon parallèle à la diminution du potentiel de base. Notons enfin que les valeurs de conductance stomatique maximales observées sur tournesol sont plus de deux fois supérieures à celles observées sur maïs $(11,5$ et $5 \mathrm{~mm} \mathrm{~s}^{-1}$ respectivement) lorsque le potentiel de base est élevé. Par ailleurs, le tournesol maintient des conductances stomatiques plus élevées que celles observées sur maïs dans une gamme de potentiel de base allant de $-0,2$ à $-1 \mathrm{MPa}$.

\section{Comparaison des effets d'une contrainte hydrique sur l'assimilation de $\mathrm{CO}_{2}$ chez le maïs et le tournesol}

La figure 4 présente l'évolution du rapport $\mathrm{A}_{n} / \mathrm{A}_{n}$ max , mesuré à midi en fonction du potentiel
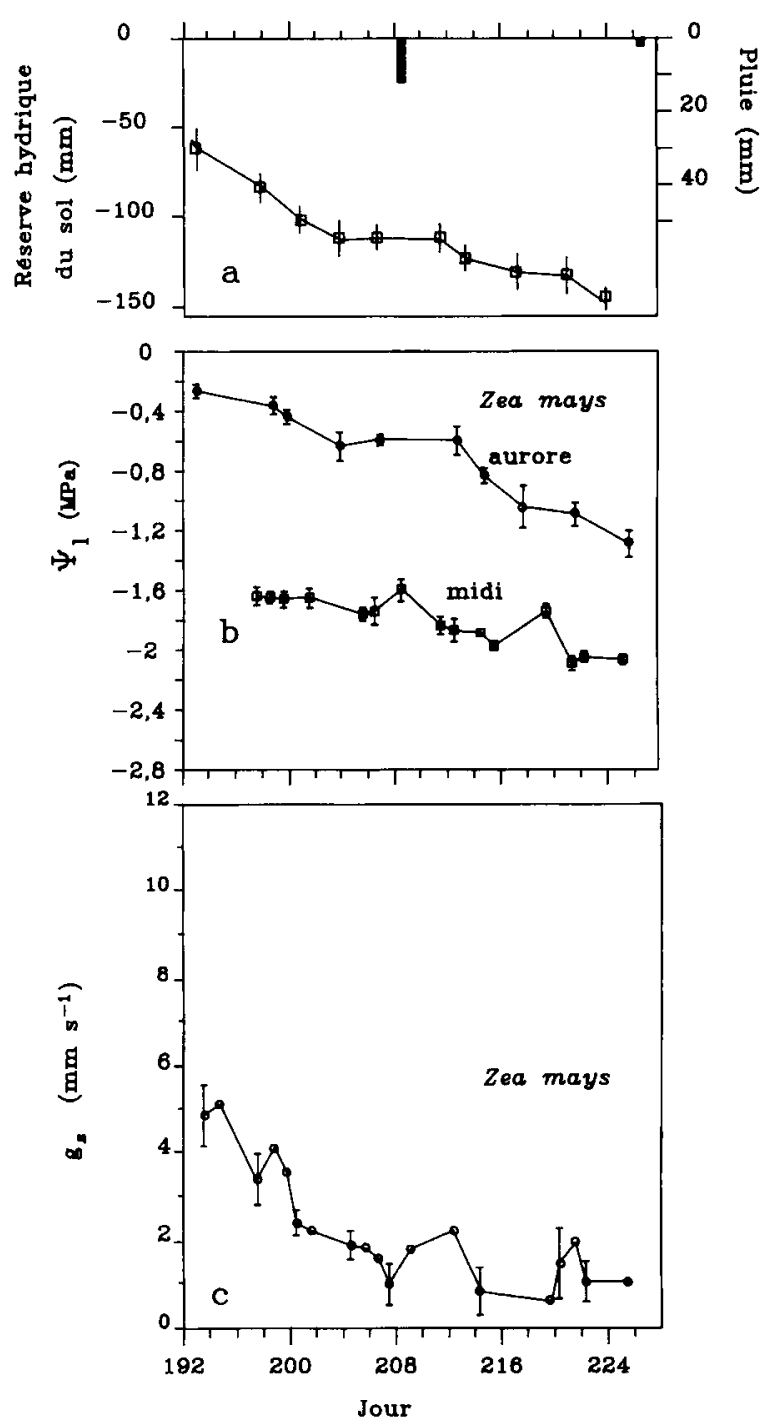

Fig 2. Modification au cours de l'été 1990 des paramètres hydriques du sol et des plantes de maïs. a. Réserves hydriques du sol et pluie. b. Potentiel hydrique foliaire $\left(\Psi_{l}\right)$ mesuré à l'aurore (potentiel de base) et à midi (potentiel minimal). Les mesures représentent la moyenne et l'écart type de six feuilles. c. Conductance stomatique pour l'eau mesurée à midi, les mesures représentent la moyenne et l'intervalle de confiance (seuil de $5 \%$ ) sur dix feuilles.

de base, chez le maïs et le tournesol. Dans ce rapport, $\mathrm{A}_{n}$ représente la valeur de la photosynthèse mesurée à midi et $\mathrm{A}_{n} \max$ la valeur maximale de $\mathrm{A}_{n}$, mesurée au début de l'expérience lorsque le potentiel de base est élevé. Cette valeur maximale varie peu au cours des trois décades qui suivent la floraison chez des plantes bien alimentées en eau (Bethenod et al, 1982), ce qui correspond à notre période d'étude. Chez le maïs, le rapport diminue rapidement avec la diminution du potentiel de base dans la gamme $-0,2$ à $-0,5 \mathrm{MPa}$. Chez le tournesol, ce rapport est à peu près constant dans une gamme de variation du potentiel de base com- 


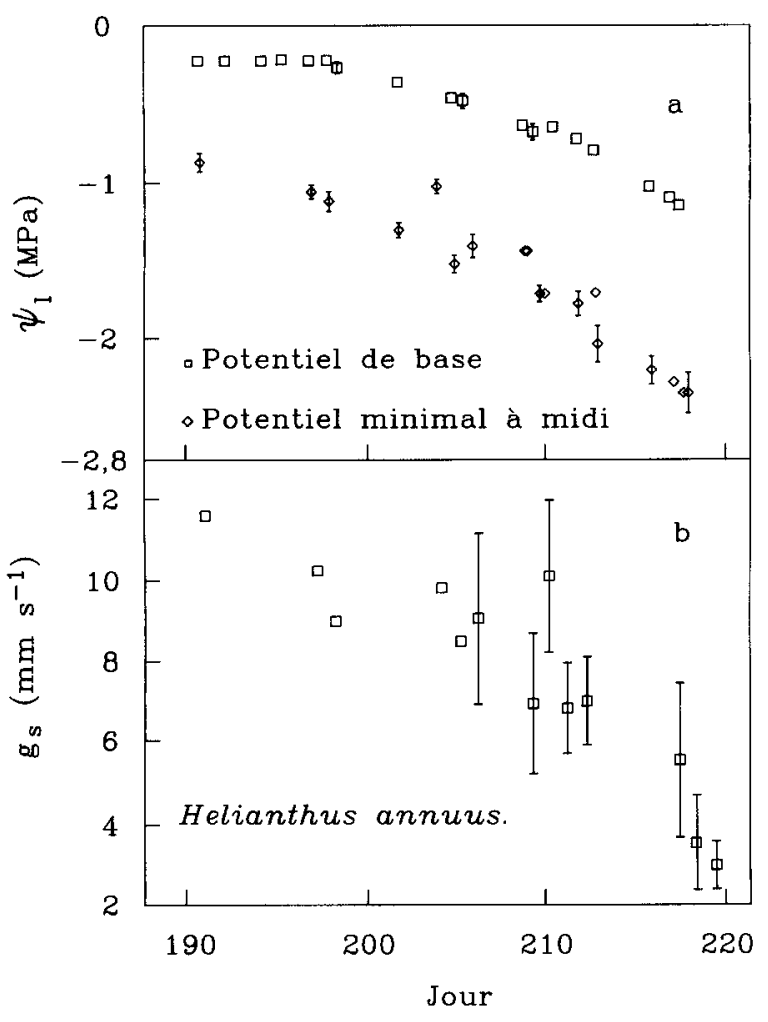

Fig 3. Modification au cours de l'été 1992 des paramètres hydriques des plantes de tournesol. a. Potentiel hydrique foliaire $\left(\Psi_{t}\right)$ mesuré à l'aurore (potentiel de base) et à midi (potentiel minimal). Les mesures représentent la moyenne et l'écart type de six feuilles. b. Conductance stomatique pour l'eau mesurée à midi, les mesures représentent la moyenne et l'intervalle de confiance (seuil de $5 \%$ ) sur dix feuilles.

prise entre $-0,1$ et $-0,7 \mathrm{MPa}$. Les valeurs basses de ce rapport sont observées lorsque que le potentiel de base devient inférieur à $-1 \mathrm{MPa}$.

\section{Efficience de l'eau chez le maïs et le tournesol en condition de contrainte hydrique}

La figure 5 présente les mesures d'assimilation nette $\left(\mathrm{A}_{n}\right)$ en fonction de la conductance stomatique pour le $\mathrm{CO}_{2}$ chez le maïs et le tournesol. La relation curvilinéaire a été ajustée à une branche d'hyperbole définie par Bethenod et al (1996) de la forme :

$$
\begin{aligned}
\mathrm{A}_{n}=\mathrm{A}_{0} & +\left[\mathrm{C} \mathrm{g}_{c}+\mathrm{A}_{n} \max -\left(\left(\mathrm{C} \mathrm{g}_{C}+\mathrm{A}_{n} \max \right)^{2}\right.\right. \\
& \left.\left.-4 \mathrm{~m} \mathrm{~A}_{n} \max \mathrm{C} \mathrm{g}_{c}\right)^{1 / 2}\right] / 2 m
\end{aligned}
$$

où $\mathrm{C}$ représente la pente initiale de la courbe, $m$ la concavité de la courbe, et $\mathrm{A}_{0}$ la valeur ajustée de $\mathrm{A}_{n}$ lorsque $\mathrm{g}_{c}$ tend vers 0 . Chez le maïs cette relation curvilinéaire entre $A_{n}$ et $g_{c}$ est proche d'une relation de proportionnalité pour des valeurs de $\mathrm{g}$. inférieure à $0,2 \mu \mathrm{mol} \mathrm{m}{ }^{-2} \mathrm{~s}^{-1}\left(R^{2}=0,881\right.$, avec 528 mesures individuelles). Il en résulte un maintien de l'efficience de l'eau selon le schéma de Jones.

Par rapport au maïs, le tournesol présente les particularités suivantes :

- l'ouverture stomatique maximale est nettement plus importante que chez le maïs ;

- pour une même valeur de conductance stomatique, la photosynthèse est nettement inférieure à celle du maïs.

En ce qui concerne la relation entre $\mathrm{A}_{n}$ et $\mathrm{g}_{c}$ chez le tournesol, elle présente une relation curvilinéaire qui s'éloigne de la proportionnalité pour les valeurs élevées de $\mathrm{g}_{c}\left(R^{2}=0,255\right.$ avec $\left.n=392\right)$, ce qui conduit aux observations suivantes. Dans la gamme de $g_{c}$ inférieure à $0,2 \mathrm{~mol} \mathrm{~m}^{-2} \mathrm{~s}^{-1}$, l'augmentation de $\mathrm{A}_{n}$ est proportionnelle à celle de $\mathrm{g}_{c}$
Fig 4. Modification du rapport $\mathrm{A}_{n} / \mathrm{A}_{n} \max$ en fonction du potentiel de base chez le maïs et le tournesol. $\mathrm{A}_{n}$ représente la moyenne sur 20 feuilles de l'assimilation nette de $\mathrm{CO}_{2}$ mesurée à midi et $\mathrm{A}_{n} \max$ l'assimilation nette maximale mesurée en l'absence de stress.

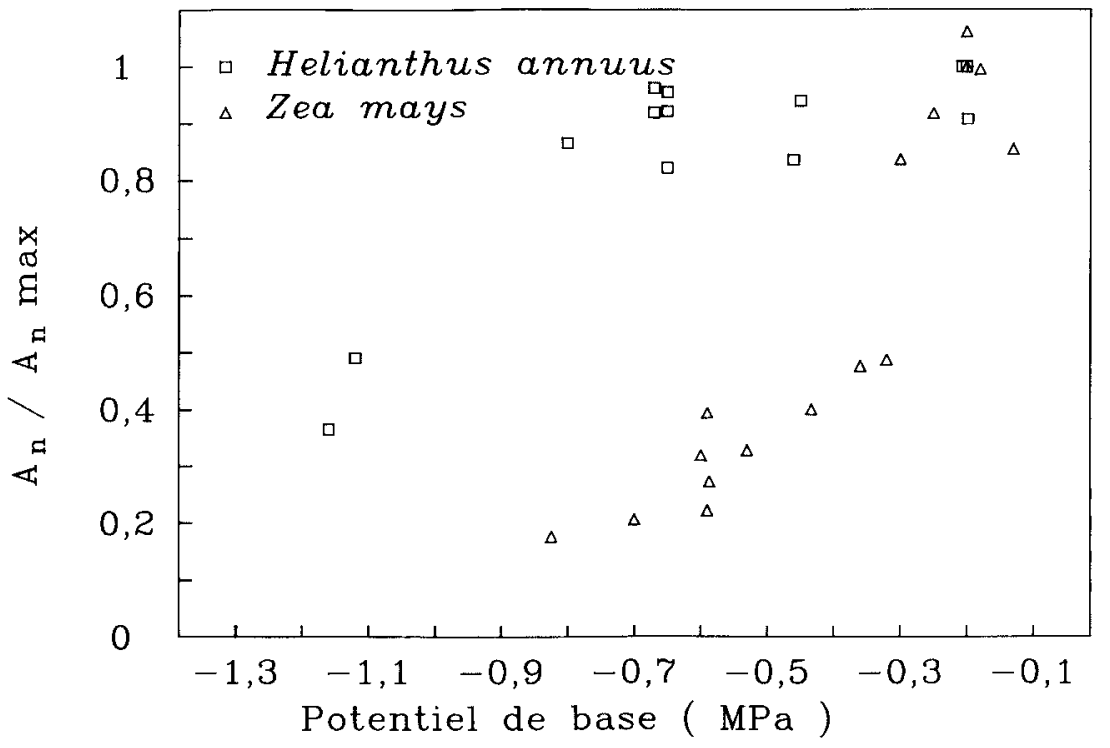




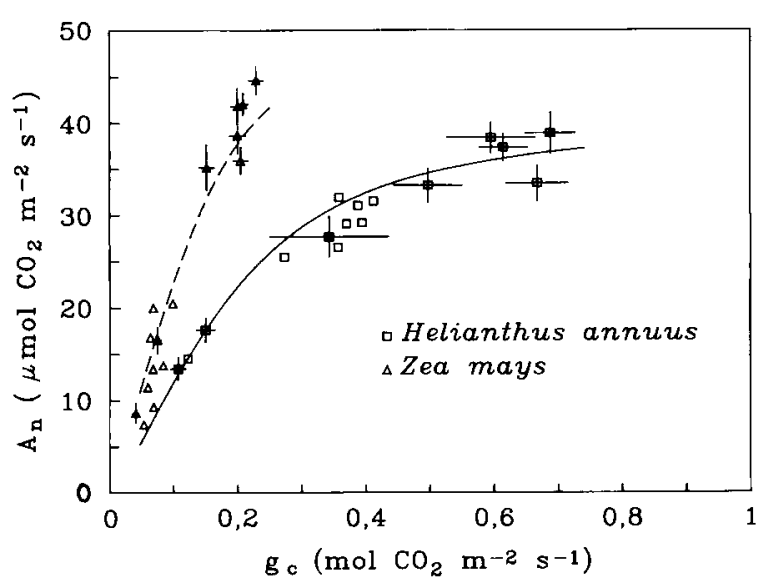

Fig 5. Relation entre l'assimilation nette de $\mathrm{CO}_{2}\left(\mathrm{~A}_{n}\right)$ et la conductance stomatique pour le $\mathrm{CO}_{2}\left(\mathrm{~g}_{\mathrm{c}}\right)$. Sont représentés la moyenne et l'intervalle de confiance (au seuil de $5 \%$ ) des mesures effectuées à midi sur 20 feuilles recevant un éclairement solaire direct supérieur à $800 \mu \mathrm{mol} \mathrm{m} \mathrm{m}^{-2} \mathrm{~s}^{-1}$. Pour la clarté de la figure, les intervalles de confiance similaires à ceux des points les plus proches ont été supprimés. Les courbes (tireté pour le maïs, trait plein pour le tournesol) ont été ajustées à une branche d'hyperbole non équilatère (Bethenod et al, 1996) de la forme :

$$
\begin{aligned}
\mathrm{A}_{n}=\mathrm{A}_{0} & +\left[\mathrm{C} \mathrm{g}_{c}+\mathrm{A}_{n} \max -\left(\left(\mathrm{Cg} \mathrm{g}_{c}+\mathrm{A}_{n} \max \right)^{2}\right.\right. \\
& \left.\left.-4 m \mathrm{~A}_{n} \max \mathrm{C} \mathrm{g}\right)^{1 / 2}\right] / 2 m
\end{aligned}
$$

avec comme valeurs des différentes grandeurs : $A_{0}$ $\left(\mu \mathrm{mol} \mathrm{m} \mathrm{m}^{-2} \mathrm{~s}^{-1}\right) \mathrm{de}-1,6$ pour les deux plantes ; $\mathrm{C}\left(\mu \mathrm{mol} \mathrm{mol}^{-1}\right)$ de 278,4 pour le maïs et 150 pour le tournesol ; $A_{n} \max$ $\left(\mu \mathrm{mol} \mathrm{m} \mathrm{m}^{-2} \mathrm{~s}^{-1}\right.$ ) de 55,6 et 43 respectivement ; $m$ (sans dimension) de 0,828 et 0,8 respectivement.

$\left(R^{2}=0,867\right.$ avec $\left.n=105\right)$. Cela se traduit par un maintien de l'efficience de l'eau, comme pour le maïs. Dans la gamme de $\mathrm{g}_{c}$ comprise entre 0,2 et $0,8 \mathrm{~mol} \mathrm{~m}^{-2} \mathrm{~s}^{-1}$, une augmentation de la conductance stomatique s'accompagne d'une augmenta-

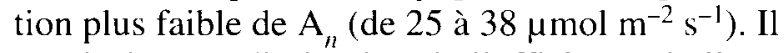
en résulte une diminution de l'efficience de l'eau selon Jones.

Notons enfin que, dans la gamme de $\mathrm{g}_{c}$ inférieure à $0,2 \mathrm{~mol} \mathrm{~m}^{-2} \mathrm{~s}^{-1}$, la pente de la relation $\mathrm{A}_{n} / \mathrm{g}_{\text {c }}$ est plus grande chez le maïs que chez le tournesol, respectivement $220 \mu \mathrm{mol} \mathrm{mol}^{-1}$ au lieu de 110 . On peut conclure que $C_{i}$ est plus élevé chez le tournesol que chez le maïs.

\section{DISCUSSION ET CONCLUSION}

Les résultats obtenus dans le cadre de cette étude apportent des informations permettant d'examiner le bien fondé des conclusions déduites de l'analyse du comportement agronomique du maïs et du tournesol et rapportées dans la littérature.
En l'absence d'une contrainte hydrique, le maïs est plus économe en eau que le tournesol et présente une efficience de l'utilisation de l'eau nettement supérieure.

Cette supériorité résulte :

- d'une conductance stomatique maximale, associée à la photosynthèse maximale, 2 à 3 fois plus réduite que chez le tournesol. Cette différence est d'ordre morphologique liée au nombre et à la taille des stomates. D'après de Parcevaux (1972), la densité stomatique est de 84 et 124 stomates au millimètre carré respectivement sur la face supérieure et inférieure des feuilles de tournesol, alors que chez le maïs elle est de moitié inférieure sur les mêmes faces ;

- d'une capacité photosynthétique élevée : il en résulte que la photosynthèse $\mathrm{A}_{n}$ est supérieure à celle du tournesol, bien que la conductance stomatique soit plus réduite. On retrouve ici une des caractéristiques des plantes de type $\mathrm{C}_{4}$ : leurs cycles biochimiques de fixation du $\mathrm{CO}_{2}$, répartis entre les chloroplastes des cellules du mésophylle et ceux des cellules de la gaine, rend plus efficace leur fonction de demande.

En condition de contrainte hydrique, le maïs adopte un comportement isohydrique (Katerji et al, 1988), c'est-à-dire qu'il régule efficacement l'ouverture stomatique tout en maintenant le potentiel hydrique foliaire dans une faible plage de variations. Le tournesol, au contraire, adopte un comportement anisohydrique : il régule faiblement l'ouverture stomatique tout en tolérant des diminutions importantes du potentiel hydrique foliaire. Dans un article précédent (Tardieu et al, 1992b), il a été démontré que les mouvements des stomates sont contrôlés par la concentration en acide abcissique du xylème. Dans ces conditions le potentiel de turgescence ne semblait pas intervenir dans le contrôle stomatique. En revanche la concentration en acide abcissique du xylème est corrélée au potentiel de base aussi bien chez le maïs (Tardieu et al, 1992a) que chez le tournesol (Tardieu et al, 1996). Chez le tournesol, la corrélation observée entre la conductance stomatique et le potentiel foliaire instantané n'est que le résultat des relations entre le flux d'eau et le gradient de potentiel dans la plante (Tardieu et al, 1996).

L'étude de l'efficience de l'eau à partir de la relation $\mathrm{A}_{n} / \mathrm{g}_{c}$ montre des différences de comportement entre le maiis et le tournesol. Chez la première espèce, il y a maintien de l'efficience de l'eau et toute diminution de l'alimentation hydrique provoque une chute de la photosynthèse. Chez le tournesol, un rationnement en eau provoque d'abord une amélioration de l'efficience de 
l'eau car la photosynthèse est faiblement affectée. Il faut attendre que la conductance se réduise à $70 \%$ environ de la conductance maximale pour qu'il y ait une proportionnalité entre $\mathrm{A}_{n}$ et $\mathrm{g}_{c}$.

Les conclusions sur le comportement du maïs et du tournesol obtenues dans le cadre de cette étude ne permettent en principe qu'une compréhension du fonctionnement instantané. Elles peuvent être introduites dans les modules du fonctionnement foliaire pour affiner les modèles de fonctionnement des cultures en condition de contrainte hydrique (par exemple Ceres maïs, Jones et Kiniry, 1986, ou QSUN, Chapman et al, 1993 et OILCROP-SUN, Villalobos et al, 1996 pour le tournesol). En revanche le caractère limité de la période d'observation rend difficile l'obtention de conclusions fiables au niveau de l'explication complète de la production.

Toutefois, il est encourageant de constater que les analyses du rendement et de consommation en eau chez le maïs et le tournesol, obtenues par l'application directe des différents seuils de potentiel hydrique de base, conduisent à des conclusions sur les efficiences de l'eau voisines de celles obtenues dans le cadre de cette étude (Ledoré et Itier, 1994).

\section{RÉFÉRENCES}

Barnes S (1938) Soil moisture and crop production under dryland conditions in Western Canada. Can Dep Agric Publ 595, 1-43

Bethenod O, Jacob C, Rode JC, Morot-Gaudry JF (1982) Influence de l'âge sur les caractéristiques photosynthétiques de la feuille de maïs Zea mays $\mathrm{L}$. agronomie 2, 159-166

Bethenod O, Katerji N, Quetin P, Bertolini JM (1988) Efficience de l'eau d'une culture de pomme de terre (Solanum tuberosum L cv Bintje). I. Mise en évidence de la régulation du $\mathrm{CO}_{2}$ interne à l'échelle foliaire. Photosynthetica 22, 491-501

Bethenod O, Tardieu F, Katerji N (1996) Relationship between net photosynthetic rate and stomatal conductance in leaves of field-grown maize subjected to soil compaction or soil drying. Photosynthetica 32, 367-379.

Blanchet R, Merrien A, Gelfi N, Cavalie G, Courtiade B, Puech J (1982) Estimation et évolution comparée de l'assimilation nette de couverts de maïs (Zea mays), tournesol (Helianthus annuus) et soja (Glycine max) au cours de leurs cycles de développement. agronomie 2, 146-154

Chapman SC, Hammer GL, Meinke H (1993) A sunflower simulation model. I. Model development. Agron J 85, 725-735 de Parcevaux S (1972) Aspects biophysiques des échanges entre les feuilles et le milieu environnant. Oecol Plant 7, 371-401

Doorenbos J, Kassam AH (1979) Yield Response to Water. FAO Irrigation and Drainage Paper 33, FAO, Rome, $193 \mathrm{p}$

Jones CA, Kiniry JR (eds) (1986) CERES-Maize. A simulation model of maize growth and development. Texas A\&M University Press, College Station, $194 \mathrm{p}$

Jones HG (1973) Limiting factors in photosynthesis. New Phytol 72, 1089-1094

Katerji N, Itier B, Ferreira I (1988) Étude de quelques critères indicateurs de l'état hydrique d'une culture de tomate en région semi-aride. agronomie 8, 425433

Katerji N, Van Hoorn JW, Hamdy A, Karam F, Mastrorilli M (1996) Effect of salinity on water stress, growth and yield of maize and sunflower. Agric Water Manag 30, 237-249

Ledoré F, Itier B (1994) Résultats de rendement suite à l'application directe de critères de stress hydrique pour le pilotage des irrigations. In : Efficient and Ecologically Sound Use of Irrigation Water With Special Reference to European Countries, 17th European Regional Conference on Irrigation and Drainage, ICID, Varna, 1, 35-46

Lesaffre B, Rieul L (1992) L'irrigation : situation actuelle et perspectives. $C R$ Acad Agric 78, 35-50

Merrien A, Grandin L (1990) Comportement hydrique du tounesol : synthèse des essais irrigation. In : Le tournesol et l'eau, Cetiom, Paris, 75-90

Mihalyfalvy I (1962) Water requirement of sunflower grown as a second crop for green manuring. Novenytermeles 11, 101-108

Penning de Vries FWT, Brunstig AHM, Van Laar HH (1974) Products, requirements and efficiency of biosynthesis: a quantitative approach. $J$ Theor Biol 45, 339-377

Puech J, Marty J, Maertens C (1976) Valorisation de l'eau consommée par les cultures en présence ou non d'irrigation. Bull Tech Inf 307, 99-103

Robelin M (1967) Action et arrière-action de la sécheresse sur la croissance et la production du tournesol. Ann Agron 18, 579-599

Robinson RG (1978) Production and culture. In: Sunflower Science and Technology, American Society of Agronomy, Madison, WI 19, 89-143

Shantz HL, Piemeisel LN (1927) The water requirements of plants at Akron. Colo J Agric Res 34, 1093 1190

Tardieu F, Katerji N, Bethenod O (1990) Relations entre l'état hydrique du sol, le potentiel de base et d'autres indicateurs de la contrainte hydrique chez le maïs. agronomie 10, 617-626

Tardieu F, Lafarge T, Simonneau T (1996) Stomatal control by fed or endogenous xylem ABA in sunflower: interpretation of correlations between leaf 
water potential and stomatal conductance in anisohydric species. Plant Cell Environ 19. 75-84

Tardieu F, Zhang J, Davies WJ (1992a) What information is conveyed by $\mathrm{ABA}$ signal from maize roots in drying field soil ? Plant Cell Environ 15, 185-191

Tardieu F, Zhang J, Katerji N, Bethenod O. Palmer S, Davies WJ (1992b) Xylem ABA controls the stomatal conductance of field-grown maize subjected to soil compaction or soil drying. Plant Cell Environ 15, 193-197

Texier V (1992) Croissance et production du tournesol dans diverses conditions de milieu. Étude expérimentale et modélisation. Thèse de doctorat, université Paul-Sabatier, Toulouse.

Villalobos FJ, Hall AJ, Ritchie JT, Orgaz F (1996) Oilcrop-Sun: a development, growth, and yield model of the sunflower crop. Agron. J. 88, 403-415 